Aus ihnen folgt für Reihe I

$G_{0}=+0.2974 \pm 0.0172$

$b=+0.0972 \pm 0.0114$

$c=+0.1262 \pm 0.0150$

$d=-0.0963 \pm 0.0337$

$e=+0.1 \mathrm{III} \pm 0.0146$

die Temperatur ist dis verbessert worden. Die erste Reihe wird jetzt wesentlich besser dargestellt als vorher, entsprechend der stark ausgesprochenen Abhängigkeit der $v_{2}$ von der Temperatur. Bei Weglassung des quadratischen Gliedes war die Fehlerquadratsumme, wie oben erwähnt, $0^{\mathrm{s}} 237^{2}$ und der mittlere Gangfehler \pm 0.0663 , während die entsprechenden Zahlen jetzt 0. I I 3 I bezw. \pm 0.5462 sind. Nicht so ausgeprägt ist die Verbesserung des Anschlusses für die zweite Reihe, bei welcher das Vorhandensein des quadratischen Gliedes ja auch nicht so stark bemerkbar war wie bei der ersten. Immerhin ist auch hier der mittlere Gangfehler kleiner geworden, wenn auch nur ganz unbedeutend. Die Zahlen waren früher $0^{s} .3469$ und \pm 0.5628 , jetzt treten an ihre Stelle 0.3203 bezw. \pm 0.0607 .

Die Zahlen der letzten Spalte in Tabelle I sind die gegen die Beobachtungen übrigbleibenden Abweichungen der mit den neuen Koeffizienten berechneten Uhrgänge. Obwohl das Zeitglied bei der letzten Auflösung der zweiten Reihe nur sehr wenig verbürgt ist, habe ich es bei der Berechnung der Gänge mit in Betracht gezogen und keine neue Ausgleichung unter Weglassung dieses Gliedes vorgenommen. Sein Einfluß auf den Uhrgang ist infolge der Kleinheit des Koeffizienten auch nur äußerst gering; er beträgt im Maximum ०.036. Der Verlauf der Fehler kann jetzt im allgemeinen als der zufälliger Beobachtungsfehler angesehen werden, obschon streckenweise gleiche Vorzeichen noch vorherrschen. Die Werte von $b$ und $c$, die vorher in den beiden Reihen recht verschieden waren, haben sich nun merklich gleichmäßiger ergeben, sodaß eine einheitliche Auflösung beider Reihen unter Konstantsetzung von $b$ und $c$ zulässig wäre. Ich schiebe diese jedoch noch auf, bis genügende weitere Beobachtungen über den Gang der Uhr vorliegen.

Göttingen, Ende April r9 6.

O. Kohl

\title{
Wirkung solarer Kathodenstrahlung in unserer Atmosphäre.
}

Schon einmal habe ich an dieser Stelle (201.247) eine kurze Mitteilung veröffentlicht über die Beziehung gesteigerter Sonnentätigkeit zu atmosphärisch-optischen Erscheinungen und damals schon die von uns besonders am i6. Juli i9 i 5 (ebenso am 3. und r9. August) beobachteten eigentümlichen Ringerscheinungen um die Sonne als Wirkung solarer Kathodenstrahlung infolge äußerst gesteigerter Fleckentätigkeit erklärt. Nun, fast genau ein Jahr später, brachten die Tage vom 2.1. bis 23. Juni I916 dieselbe Erscheinung wieder bei Anlaß der nämlichen Begleitumstände auf der Sonne.

Nach gefälligen Mitteilungen von Herrn Professor $W o l f e r$, Direktor der Eidg. Sternwarte, fand am 21. Juni I9 I 6 der Eintritt eines ausgedehnten Gebietes mit sehr lebhafter Fleckentätigkeit statt und zwar gleichzeitig auf der nördlichen und südlichen Sonnenhalbkugel. Am 21 . und 22. wurden auch zahlreiche metallische Protuberanzen beobachtet, und die Epoche charakterisierte sich überhaupt durch beständige Neubildungen und maximale Fleckentätigkeit. Seit den Tagen von Mitte Juni I9I5 war sie die stärkste; es gingen die relativen Fleckenzahlen bis ca. I80. Am 2 I. Juni nachmittags zeigte die markante Dunstscheibe um die Sonne eine äußerst starke Anschwellung bis auf ca. $120^{\circ}$ Durchmesser, deutlich beobachtet auf der Toggenburger Höhenwarte in Oberhelfenswil ( $900 \mathrm{~m}$ ï. d. M.). Die äußere Umrandung der großen Dunstscheibe hatte gleichzeitig einen zarten, bräunlichen Stich; am 22 . und 23. Juni wiederum bei auffallend starker Dunstkorona von mindestens $100^{\circ}$ Durchmesser dieselbe bräunliche Umsäumung.

Gleichzeitig stellte mir Herr Dr. Dorno, Davos-Platz, in liebenswürdigster Weise sein wertvolles Material aus diesen kritischen Tagen ebenfalls zur Verfügung, aus dem mit Sicherheit hervorging, daß bereits am I9. Juni, vielleicht schon am I 7., eine atmosphärisch-optische Störung in Begleitung der stark gesteigerten Sonnentätigkeit vorhanden war, daß sie am 22. vorïbergehend etwas abschwächte und bezüglich der solaren Dunstscheibe ähnlich abklang wie seinerzeit die große Katmai-Störung, indem der innere Ring sich auf die Sonne zurückzog, der äußere dagegen sich weit ausdehnte und im Himmelsblau verlief.

Vom meteorologisch-magnetischen Observatorium in Potsdam erhielt ich die gefällige Mitteilung, daß "vom 2 I. bis 23. Juni mäßige magnetische Unruhe vorhanden war". Nordlicht wurde, soweit ich bis jetzt in Erfahrung bringen konnte, nirgends beobachtet.

Aus dem Vorstehenden mag wiederum hervorgehen, daß zu gewissen Zeiten bei gesteigerter Fleckentätigkeit infolge Kathodenstrahlenwirkung von der Sonne her, besondere Ringerscheinungen um letztere entstehen. Wir werden der Angelegenheit auch weiterhin unsere volle Aufmerksamkeit schenken. Zürich, im Juli r 9 r 6.
F. Maurer.

\section{Mitteilung betreffend die Zustellung der Astronomischen Nachrichten.}

Die aus den gegebenen Verhältnissen hervorgehende Unsicherheit der Verkehrsverhältnisse bringt es leider mit sich, daß manchen Beziehern der Zeitschrift die einzelnen Nummern unregelmäßig zugehen oder vorläufig ganz ausbleiben. Der Herausgeber, der diesen Verhältnissen machtlos gegenübersteht, kann nur um Nachsicht bitten. Da aber sicher anzunehmen ist, daß viele der jetzt nicht eingehenden Nummern später sich doch noch einstellen werden, und andererseits eine Nachlieferung jetzt, wenn sie nochmals von Verlust betroffen werden sollte, wegen der beschränkten Anzahl der vorhandenen überzähligen Abzüge die Gefahr hervorrufen würde, daß ein Ersatz schließlich unmöglich wäre, hält der Herausgeber es für geboten, Nachforderungen erst zu erledigen, wenn sie mit Sicherheit ausgeführt werden können. Der Herausgeber.

Inhalt zu Nr. 4854, L. Courvoisier. Über das mehrfache System des Polarsterns. 85. - O. Kohl. Der Gang der Hauptuhr der Göttinger Sternwarte, Dencker Nr. 35. 91. - 7. Maurer. Wirkung solarer Kathodenstrahlung in unserer Atmosphäre. 99. Mitteilung betreffend die Zustellung der Astronomischen Nachrichten. 99. 